\title{
An In-Band Full-Duplex Transceiver for Simultaneous Communication and Environmental Sensing
}

\author{
Seyed Ali Hassani ${ }^{1}$, Andrea Guevara ${ }^{1}$, Karthick Parashar ${ }^{2}$, André Bourdoux ${ }^{2}$, Barend van Liempd ${ }^{2}$ and Sofie Pollin ${ }^{1}$ \\ ${ }^{1}$ Department of Electrical Engineering, KU Leuven, Belgium \\ ${ }^{2}$ Imec, Leuven, Belgium
}

\begin{abstract}
To obtain Tx-Rx isolation in an in-band full-duplex (IBFD) transceiver, the electrical-balance duplexer (EBD) can be utilized to suppress the self-interference (SI). Although the EBD can provide more than $50 \mathrm{~dB}$ cancellation of the SI directly at RF, it cannot fully overcome strong signals reflecting off of nearby objects. As a result, the environmental reflections could still limit the performance of IBFD communication and have to be attenuated in an extra cancellation step, e.g. in digital baseband using a digital SI canceler. While mitigating the reflections is a challenging dimension of this technology, it opens a new opportunity to perform environmental sensing. In this paper, an IBFD transceiver architecture is introduced to enable such context-aware communication functionality. We investigate how the EBD's SI rejection property influences the performance of the proposed communication device which also functions as a Doppler radar. The simulation result demonstrates that the proposed architecture can produce high-resolution Doppler when the EBD provides $>20 \mathrm{~dB} \mathrm{Tx}-\mathrm{Rx}$ isolation. Concerning the constraints dictated by the application, this paper finally suggests a radar-communication trade-off.
\end{abstract}

Index Terms-In-band full duplex, self-interference cancellation, Doppler radar, wireless sensing.

\section{INTRODUCTION}

With the recent explosion of wireless devices and to quench the ever-increasing demand for higher data rates, the spectrum utilization has to be further enhanced in the wireless communication domain. In-band full duplex (IBFD) technology has been identified as a promising solution to overcome this challenge as it enables concurrent transmit and receive over the same frequency band. Significant interest exists in rearchitecting terrestrial wireless communications systems, such as Wi-Fi and cellular networks, as a way of leveraging IBFD transmission [1].

Improving spectral efficiency and throughput, however, is not the only advantage of IBFD. In the last few years, this technology has also attracted attention as a potential solution to overcome other communication and networking challenges such as in-band relaying [2] and the hidden terminal issue [3], as it resolves the deafness in transmitting period. In this context, IBFD can also be employed to integrate wireless sensing into a communication device. Basically, in full duplex schemes, the device can recapture the environmental reflections of the signal it transmits and interprets the physical dynamics in the vicinity.
Environmental sensing by a wireless communication device has been intensively investigated in the literature in the form of opportunistic passive wireless sensing systems where the ambient electromagnetic signal is used to extract the physical motion information. This sensing capability can find applications widely ranged from body and hand gesture recognition to e-healthcare, localization and through-the-wall tracking. The authors in [4]-[7] introduce techniques to perform localization, and human activity gesture detection via estimating the received signal strength indicator (RSSI) by leveraging the WiFi signal. This approach, however, is sensitive to the influence of the physical environment as the RSSI significantly depends on the temporal and spatial variance due to the multipath effect [8].

Recent attempts in opportunistic wireless sensing have focused on the extraction of Doppler frequency shifts. Generally, a specific pattern of motions generates a particular set of Doppler frequencies, a so-called Doppler signature, which can be learned by a classifier to perform applications such as hand gesture recognition. The authors in [9]-[11] present bistatic passive radar systems where one or multiple receivers are employed to obtain the Doppler information from the ambient Wi-Fi signal. This passive scheme requires directional antennas to collect the reference and surveillance signals separately. The former is the direct signal illuminated by the source, a Wi-Fi access point (AP) for instance, and the latter is the reflected signal from the target. This approach estimates the Doppler frequency by mixing the reference and surveillance signals.

Apart from the technical implementation difficulties such as synchronization, the performance of bi-static passive radars is always sensitive to the isolation of the reference channel. To overcome this issue, these systems use beam antennas which limit the system angle of view (AOV). To extend the AOV hence, multiple directional surveillance receivers has to be employed [12].

This paper introduces a context-aware IBFD transceiver which allows simultaneous communication and environmental sensing. The proposed system benefits from the state-of-the-art analog duplexer to render concurrent $\mathrm{Tx}$ and $\mathrm{Rx}$ operation in full duplex mode. Thus, by integrating the surveillance receiver into the illuminator, one can avoid the sensitive process of 


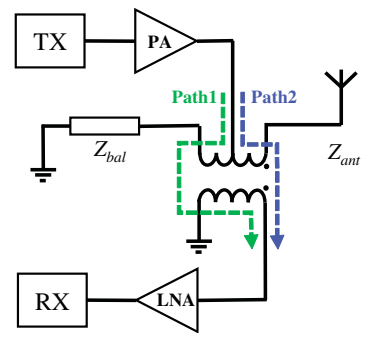

Fig. 1. An exemplary illustration of electrical balance duplexer (EBD).

over-the-air reference signal extraction. Besides, this approach significantly simplifies implementation as no signal processing is needed to estimate the reference signal. The analog duplexer, however, dictates particular functioning conditions which can introduce distortion and limit Doppler detection. In this paper, we investigate various distortion sources which influence the radar performance of the proposed system and study the effective velocity detection range when the Doppler frequency shift is extracted from each single Tx packet.

The remainder of this paper is structured as follows. Next section presents the proposed IBFD-based context-aware communication system, expands a signal model and formulates the system. Section III employs the model to investigate its behavior in a simulated scenario. Section IV discusses the radar and communication performance trade-off and finally, the conclusion is drawn.

\section{RADAR-CAPABLE IBFD COMmUNiCATION DEVICE}

This section firstly reviews the concept of the electrical balance duplexer (EBD) as the essential element of the proposed context-aware transceiver and then the system architecture is established and formulated.

\section{A. Self-interference Cancelation by EBD}

Concurrent $\mathrm{Tx}-\mathrm{Rx}$ operation exposes the receiver to the strong signal leaking from the paired transmitter, so-called self-interference (SI). This interference has to be suppressed substantially at RF before it saturates the receiver chain and wastes the dynamic range of the analog to digital converters (ADCs). Thus, the analog self-interference cancelation (AnSIC) stage plays an essential role as it facilitates a complementary SI rejection step after ADC sampling by the digital self-interference cancelation (DiSIC) module.

Various AnSIC approaches have been proposed so far, some of which achieve better than $70 \mathrm{~dB}$ Tx-Rx isolation [13]. The EBD is a recently introduced AnSIC solution that reveals interesting properties. Thanks to silicon-on-insulator technology, the EBD can be deployed on-chip and this feature suits it for mobile communication devices. In addition to its small size, high linearity and low insertion loss, the EBD also allows a broad center frequency tuning range that privileges it rather than the fixed-frequency counterparts, e.g., surfaceacoustic-wave (SAW) filters.

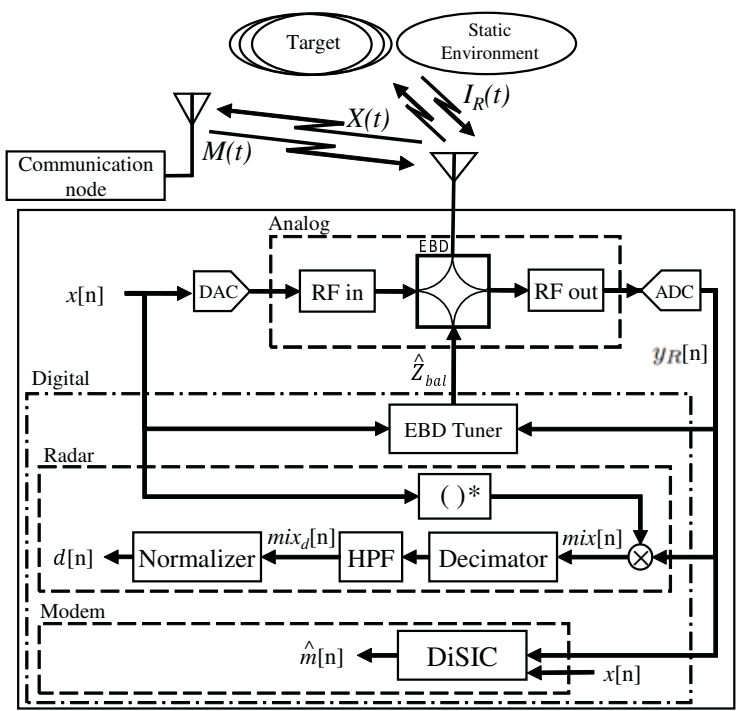

Fig. 2. Architecture of the proposed radar-capable IBFD transceiver.

Fig. 1 depicts the representation of an EBD comprising two main components; a hybrid transformer coupled with a tunable impedance network $Z_{b a l}$. The four terminals of the transformer are connected to the transmitter and receiver, as well as the antenna and the $Z_{b a l}$. Applying the ideal S-parameters of a symmetric hybrid transformer $(r=1)$, the isolation can be obtained as follows:

$$
\begin{gathered}
I_{T x-R x}=20 \log _{10}\left|\Gamma_{a n t}-\Gamma_{b a l}\right|-6.02, \\
\Gamma_{a n t}=\frac{Z_{a n t}-R_{o}}{Z_{a n t}+R_{o}} \text { and } \Gamma_{b a l}=\frac{Z_{b a l}-R_{o}}{Z_{b a l}+R_{o}},
\end{gathered}
$$

where $R_{o}$ is the output load and $Z_{a n t}$ is the antenna impedance. According to (1), when $\Gamma_{a n t} \cong \Gamma_{b a l}$, high isolation can be achieved because the two equivalent copies of the SI signal (through Path 1 and Path 2 in Fig. 1) reach the receiver port in opposite phase. Therefore, to deliver wideband Tx-Rx isolation, $\Gamma_{a n t}$ and $\Gamma_{b a l}$ either have to be frequency independent or reveal the same frequency dependence [14].

Additionally, the antenna impedance varies by the dynamics in the environment and hence, an adaptive tuning algorithm has to be embedded into the full duplex system to track the environmental changes and generate optimum $Z_{b a l}$ coefficients.

\section{B. System Model}

Fig. 2 shows the block diagram of the proposed IBFD transceiver in which an EBD-based AnSIC is employed to provide the essential Tx-Rx isolation. Through feedback from the EBD receiver port in this design, a tuner can measure the transmitter leakage and balance $Z_{a n t}$ and $Z_{b a l}$ adaptively to minimize the SI. The system also benefits from a DiSIC module to overcome the residual SI signal and enable communication functionality by estimating the desired signal. 
In this figure, $I_{R}(t)$ is the environmental reflections of the transmit RF signal $X(t)$ and $M(t)$ represents the intended signal coming from a second party transceiver. This section develops the system model in the baseband, in which the notations $i_{R}[n], x[n]$ and $m[n]$ are the discrete representation of the RF signals introduced above, after downconversion and complex sampling. The signal $\hat{m}[n]$ also presents the approximated desired signal by DiSIC block.

The transmit signal $x[n]$ can be presented as a complex signal in terms of time-varying magnitude and phase, i.e.,

$$
x[n]=A_{x}[n] e^{j \varphi_{x}[n]},
$$

where $A_{x}[n]$ and $\varphi_{x}[n]$ determine the amplitude and phase modulations respectively.

For brevity, we simplify the notations in the rest of the paper by dropping $[n]$ from the signals that are previously introduced within an explicit equation.

The reflection of the transmitted signal arrives at the receiver through multiple paths. Employing the channel model in [15], we describe the baseband representation of the reflection $i_{R}[n]$ as a sum of echoed SI from static and dynamic paths in the form

$$
i_{R}[n]=i_{R, s}[n]+i_{R, d}[n],
$$

where $i_{R, s}[n]$ stands for the reflection of the static environment and $i_{R, d}[n]$ represents the mirrored signal from the moving target.

The static reflection can be expressed as an aggregation of attenuated and phase shifted copies of the transmit signal $x$, i.e.,

$$
i_{R, s}=\sum_{k \in P_{s}} A_{k} e^{j \varphi_{k}} x,
$$

where $P_{s}$ is the set of dominant reflection paths caused by the static objects in the surroundings, $A_{k}$ represents the constant attenuation for the $k^{t h}$ path with the initial phase offset of $\varphi_{k}$.

For the sake of simplicity, it is also assumed that there is a single varying path caused by the moving target. Accordingly, the reflected SI component through the dynamic path can be written as follows:

$$
i_{R, d}=A_{d}[n] e^{j\left(w_{d}[n]+\varphi_{d}\right)} x
$$

where $A_{d}[n]$ is the attenuation of the dynamic path with an initial phase of $\varphi_{d}$ and $w_{d}[n]$ is the Doppler frequency shift we are interested in. Note that due to the small signal bandwidth and small operational range of the applications targeted by this paper ${ }^{1}$, the phase difference between the transmit signal and its reflections is assumed constant over the signal bandwidth.

To model the AnSIC in the proposed system, we represent the EBD as a band-stop filter by a complex-valued SI rejection factor $g_{e}$. Accordingly, the remaining direct SI component $i_{S}[n]$ in the digital baseband can be written as

${ }^{1} \mathrm{BW}<20 \mathrm{MHz}$ and Range $<10 \mathrm{~m}$

$$
i_{S}[n]=g_{e} x,
$$

where $g_{e}=G_{e} e^{j \varphi_{e}}$ and, $G_{e}$ and $\varphi_{e}$ are respectively the magnitude and phase of the EBD SI cancelation factor.

In a realistic operating condition, the received signal $y_{R}[n]$ in the baseband before digital SI cancelation comprises three main components: the residual direct SI $i_{S}$, the reflections $i_{R}$ and the desired message $m$. Thus, this signal can be expressed as

$$
y_{R}[n]=i_{S}+g_{e, r}\left(i_{R}+m\right),
$$

where $g_{e, r}=G_{e, r} e^{j \varphi_{e, r}}$ is the complex-valued representation of the imposed attenuation and phase shift by the EBD to the signals that the antenna receives. It should be noted that the EBD parameters, i.e., $g_{e}$ and $g_{e, r}$, are constant as long as the EBD's impedance network is unchanged.

As shown in Fig. 2, the reflection $I_{R}(t)$ can be seen as the surveillance signal which carries the radial velocity information of the target. However, unlike the bi-static passive radar, the device has direct access to the interference-free reference signal $x$. To extract the Doppler shift hence, $y_{R}$ is mixed with the complex conjugate of the reference resulting in the following expression.

$$
\operatorname{mix}[n]=x^{*} y_{R}=x^{*}\left(i_{S}+g_{e, r}\left(i_{R, d}+i_{R, s}+m\right)\right),
$$

where (*) is the complex conjugate operation, (shown by ()$^{*}$ in the block diagram).

In the context of Doppler radar, the complex multiplication of the conjugated transmit signal and its reflection from the target, the term $x^{*} g_{e, r} i_{R, d}$ in (9), yields the Doppler information. However, there are three sources of distortion in the estimated mixed signal: the residual direct SI $i_{S}$, the reflection caused by the static objects $i_{R, s}$, and the intended message $m$. Next, this sub-section explains how the proposed system compensates the interfering components in the mixed signal to enable Doppler extraction.

The distortion caused by the residual direct SI $\left(x^{*} i_{S}\right)$ is immediately influenced by the magnitude of the EBD SI rejection factor $G_{e}$. Therefore, providing adequate SI cancelation at RF can significantly reduce the impact of this distortion.

Besides, by employing the definitions presented in (3), (5) and (7), it can be shown that the distortion caused by the residual direct SI $\left(x^{*} i_{S}\right)$ and the interference introduced by the static objects $\left(x^{*} g_{e, r} i_{R, s}\right)$, produce zero-frequency I-Q vectors after decimation, as long as $x$ is a zero-mean signal. To eliminate these unwanted elements, a DC-removal filter, shown by HPF block in Fig. 2, is embedded in the proposed IBFD transceiver.

The low-pass filtering in the decimation procedure also can suppress the distortion of the desired message, i.e., the term $x^{*} g_{r, e} m$ in (9), as $m$ is unlikely to be correlated with $x$.

Therefore, assuming that the EBD provides adequate SI rejection and considering that $x$ and $m$ are uncorrelated, we 
can approximate the decimated and DC-removed mixed signal $\operatorname{mix}_{d}[n]$ as follows:

$$
\operatorname{mix}_{d}[n] \cong x^{*} g_{e, r} i_{R, d}=A[n] e^{j\left(w_{d}[n]+\varphi_{d}+\varphi_{e, r}\right)},
$$

where $A[n]$ is the amplitude of the decimated mixed signal. Given the baseband sample rate $f_{s}$ and the decimation factor $N_{d}$, mix $x_{d}$ streams at $f_{s} / N_{d}$. To remove the amplitude modulation, this signal is normalized resulting in a narrow-band Doppler signal given in (11)

$$
d[n]=\frac{\text { mix }_{d}}{\left|m i x_{d}\right|}=e^{j\left(w_{d}[n]+\varphi_{d}+\varphi_{e, r}\right)} .
$$

\section{Doppler Velocity Resolution and Boundaries}

Applying the fast Fourier transform (FFT) in the next step, we can analyze the Doppler signal $d$ to estimate the realvalued instantaneous Doppler frequency $f_{d}(H z)$ and the instantaneous velocity of the target $v(\mathrm{~m} / \mathrm{s})$.

$$
f_{d}=2 \frac{v}{c} f_{c},
$$

where $c$ is the speed of light and $f_{c}$ is the carrier frequency.

Accordingly, the velocity resolution $v_{r e s}$ and the maximum detectable velocity $v_{\max }$ can be determined as follows:

$$
\begin{gathered}
v_{r e s}=\frac{1}{2} \frac{c}{f_{c}} \frac{f_{s}}{N_{d} N_{f f t}}, \\
v_{\max }=\frac{1}{4} \frac{c}{f_{c}} \frac{f_{s}}{N_{d}},
\end{gathered}
$$

where $N_{f f t}$ is the number of points used to obtain the FFT.

As it can be seen in (11), the EBD's impedance network has to remain unchanged during Doppler detection since it imposes a post-tuning phase component $\varphi_{e, r}$ to the detected Doppler signal. Therefore, the minimum detectable velocity and consequently, the detectable velocity range $\left[v_{\min }, v_{\max }\right]$ are limited to the $Z_{b a l}$ update rate $f_{\text {tune }}$, i.e.,

$$
v_{\text {min }} \geq \frac{1}{2} \frac{c}{f_{c}} f_{\text {tune }} .
$$

\section{Simulation Results}

This section aims to: a) validate the approximation used in (10) to estimate the decimated mixed signal and b) evaluate the environmental sensing capability of the proposed model. To this end, we chose an OFDM waveform for both the transmit signal $x$ and the desired message $m$, using parameters as specified in Table I for IEEE 802.11. The simulation is carried out in Matlab in double precision floating-point, the in-band thermal noise is assumed to be $-90 \mathrm{dBm}$ and the IBFD receiver samples at $40 \mathrm{MHz}$. The transmit signal and the message $m$ are modeled by two statistically independent and zero-mean random data streams. The simulator computes the baseband received signal taking into account the introduced components in (8) to obtain the interfered mixed signal in (9). The decimated mixed signal is then estimated at $610 \mathrm{~Hz}$,
TABLE I

IEEE 802.11-LIKE OFDM WAVEFORM CHARACTERISTICS

\begin{tabular}{|c|c||c|c|}
\hline Param. & Value & Param. & Value \\
\hline strength & $20 \mathrm{dBm}$ & OFDM subcarriers & 52 \\
\hline bandwidth & $20 \mathrm{MHz}$ & OFDM pilots & 4 \\
\hline bit rate & $9 \mathrm{Mbps}$ & modulation & QPSK \\
\hline
\end{tabular}

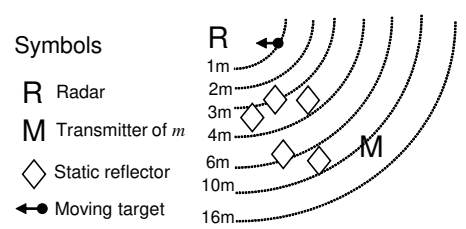

Fig. 3. Layout of the simulated multipath channel.

filtered, normalized and windowed (Hanning) to generate FFT components. This configuration enables up to $\pm 19 \mathrm{~m} / \mathrm{s}$ velocity measurement and the velocity resolution of $12.4 \mathrm{~cm} / \mathrm{s}$ for an observation window of $500 \mathrm{~ms}$.

Fig. 3 depicts the simulated multipath scenario where five static reflectors are positioned in $3-12 \mathrm{~m}$ around the proposed IBFD transceiver to resemble a multipath channel. In addition to the transmitter of the desired signal $m$, a moving target is also involved to produce Doppler frequency shifts. In this section, the speed and distance of the target, relative to the IBFD transceiver, are denoted by $v_{T}$ and $d_{T}$ respectively. The notation $d_{m}$ is also adopted to present the distance of the transmitter of $m$ from the proposed device.

As illustrated in Fig. 4 (Left), in the first test, the target moves away from the device at constant acceleration. Once it reaches in $4.4 \mathrm{~m}$, decreases the speed until it stops at $7 \mathrm{~m}$ and returns toward the device with the same acceleration. The EBD SI suppression factor is also set to $-50 d B$ to provide constant SI cancelation in this test. Fig. 4 (Right) shows the estimated Doppler profile. This graph confirms that the system can produce an accurate Doppler profile which indicates the speed of a target moving in various distances.

In the next test, we evaluate the velocity estimation performance of the system in the form of the signal to noise ratio (SNR) of the estimated Doppler signal $S N R_{d}$, in which the signal power is measured in the range of $\pm 2 f_{s} / N_{d} N_{f f t}$ around the expected Doppler frequency component. Fig. 5 (Left) shows $S N R_{d}$ for different EBD SI cancelation levels when the transmitter of the desired signal is located at different distances $\left(d_{m}=16,10,7\right.$ or $\left.5 \mathrm{~m}\right)$ and a target situated $2 \mathrm{~m}$ away from the device is moving at $2 \mathrm{~m} / \mathrm{s}$. This simulation shows that increasing the analog SI rejection enhances the radar performance. Most importantly, this simulation validates the approximation in (10) that given $x$ and $m$ are indeed uncorrelated, the interference from the desired signal is insignificant on the Doppler estimation performance. Besides, this graph proves that the DC-removal filter after decimation significantly suppresses the distortions created by the static objects and the residual SI. 

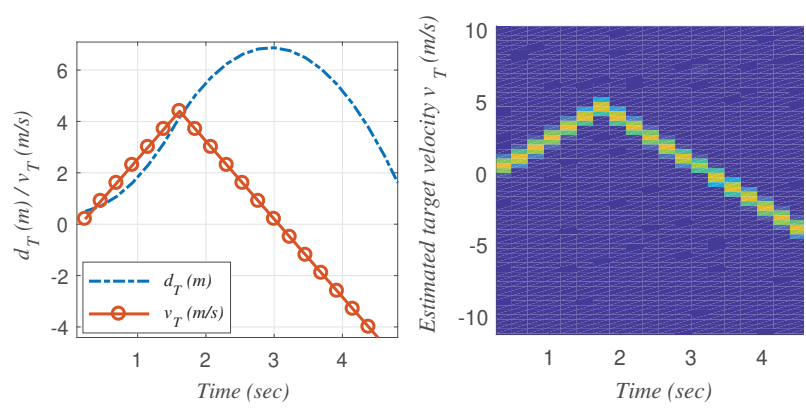

Fig. 4. (Left) The distance and the radial speed of the simulated target, i.e. $d_{T}$ and $v_{T}$ respectively. (Right) Estimated Doppler profile for a target moving in a simulated scenario with constant analog SI rejection $\left(G_{e}=-50 \mathrm{~dB}\right)$.
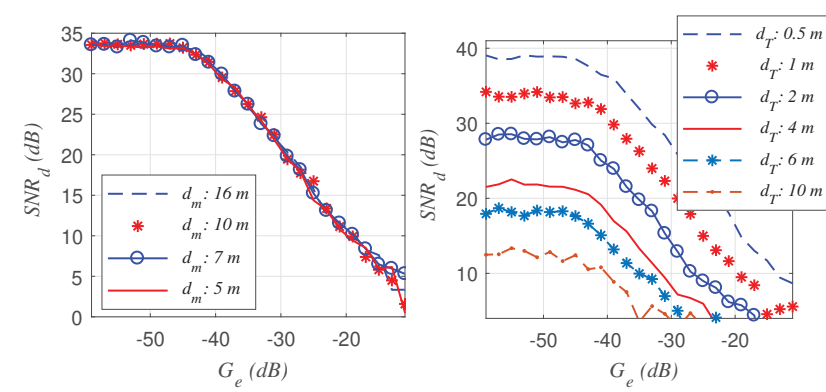

Fig. 5. Signal to noise ratio of the estimated Doppler signal as a function of the magnitude of the EBD SI rejection factor $G_{e}$; (Left) when the transmitter of the desired signal $m$ is located at different distance $\left(d_{m}=16,10,7,5 \mathrm{~m}\right)$ from the IBFD device. (Right) when the target moves at different ranges within $0.5-10 \mathrm{~m}$.

Fig. 5 (Right) indicates $S N R_{d}$ for targets at different distances from the proposed IBFD transceiver while the transmitter of the desired signal is always in $16 \mathrm{~m}$. This figure demonstrates how $S N R_{d}$ is degraded by increasing the distance of the target, however, improving the EBD's SI rejection increases the quality of the estimated Doppler.

\section{RADAR-COMMUNICATION TRADE-OFF DisCUSSION}

The proposed device is assumed to allow in-band transmission and reception and simultaneous measurement of the surrounding changes in the form of the Doppler profile. While the EBD provides significant Tx-Rx isolation, it requires frequent $Z_{b a l}$ tuning. Thus, to obtain both aimed functionalities, the adaptation algorithm has to be triggered under certain conditions:

- The EBD tuning includes a feedback mechanism which measures the amount of the residual SI. Consequently, the tuning process has to be accomplished during data transmission.

- Setting $Z_{b a l}$ iteratively in the EBD adaptation procedure induces a phase distortion at the EBD receiver terminal (see $g_{e, r}$ in (8)). As a result, the system has to avoid the EBD re-tuning in the receiving cycle.

Since the both send and receive operations are controlled by the medium access control (MAC) layer, the EBD re-
TABLE II

Packet-Wise EBD tuning, Minimum Detectable Velocity

\begin{tabular}{|c|c|c|}
\hline Waveform. & Max Packet Duration $T_{t x}$ & $v_{\min }$ \\
\hline Zigbee, $868 \mathrm{MHz}, 20 \mathrm{kbps}$ & $53 \mathrm{~ms}$ & $3.2 \mathrm{~m} / \mathrm{s}$ \\
\hline Zigbee, $915 \mathrm{MHz}, 40 \mathrm{kbps}$ & $26.6 \mathrm{~ms}$ & $6.2 \mathrm{~m} / \mathrm{s}$ \\
\hline Wi-Fi, $2.4 \mathrm{GHz}, 1 \mathrm{Mbps}$ & $1.1 \mathrm{~ms}$ & $62.2 \mathrm{~m} / \mathrm{s}$ \\
\hline
\end{tabular}

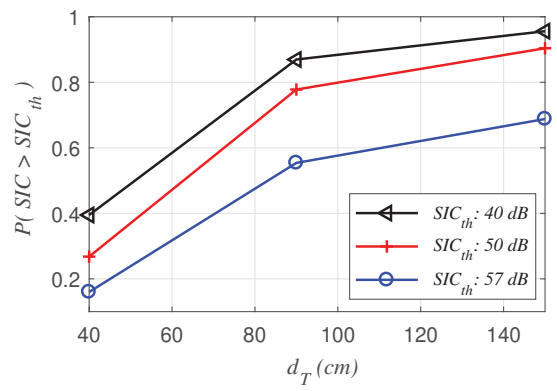

Fig. 6. Measured probability that the analog SI cancelation level $S I C$ is better than a given threshold $S I C_{t h}$, when a target moves in different distances $d_{T}$.

tuning also has to be handled by the MAC layer. In the next subsection, we study packet-wise EBD tuning scheme to see how to trade off system performance for joint sensing and communication.

\section{A. Packet-wise EBD Tuning}

In the packet-wise EBD tuning strategy, the tuner performs adaptation at the Tx packet startup. Although the minimum detectable velocity $v_{\min }$ in this method is limited to the packet length $T_{t x}$, maximum EBD performance is guaranteed by frequent tuning. Therefore, the practical approximation of $v_{\text {min }}$ in (15) can be written as

$$
v_{\text {min }} \geq \frac{1}{2} \frac{c}{f_{c}} \frac{1}{\left(T_{t x}-T_{\text {tune }}\right)},
$$

where $T_{\text {tune }}$ represents the time that the EBD tuning algorithm requires to estimate the optimum coefficients $\hat{Z}_{b a l}$.

By employing this tuning approach, Table II lists $v_{\text {min }}$ for a few communication standards, assuming that the EBD can be tuned within $0.1 \mathrm{~ms}$. This table suggests that applications with slower physical dynamics are feasible with low data rate standards.

An interesting question that arises here is to what extent the need for EBD re-tuning depends on the distance of the target. Understanding this connection helps to propose a maximal packet length, influencing the EBD tuning frequency and consequently, the detectable velocity range. To answer this question, we set up a measurement campaign which determines the probability that the overall analog SI cancelation $S I C$ is higher (better) than a pre-defined cancelation threshold $S I C_{t h}$ during the length of the transmit packet $T_{t x}$. If the SI suppression is lower than this threshold, the communication performance will be impacted by the high SI. In this measurement, an XY table is used to move a reflector, back and forth 
at the speed of $4 \mathrm{~cm} / \mathrm{s}$ in various distances from our EBDequipped software defined radio (SDR) [16] which employes a dithered linear search (DLS) optimizer [17] to tune the EBD. Given the threshold $S I C_{t h}$, the probability $P\left(S I C>S I C_{t h}\right)$ is then measured within $60 \mathrm{~s}$ after an initial EBD tuning. The measurement result in Fig. 6 illustrates that by increasing the target distance the SI rejection level is more likely to satisfy the threshold constraint and hence, less EBD re-turning would be needed. Furthermore, the higher the threshold, the more frequently the SI signal has to be suppressed to achieve a better communication performance. However, a higher $S I C_{t h}$ also allows shorter $T_{t x}$ packets and hence bounds the radar performance to detection of high speeds.

\section{Conclusion}

This paper presents an IBFD transceiver which simultaneously senses the environmental dynamics in the form of a Doppler frequency profile. Comparing with the traditional bi-static Doppler radar systems, our solution does not suffer from synchronization difficulties and noisy reference signal as it benefits from a co-located Tx and Rx which operate concurrently. To overcome the self-interference in the presented design, we have utilized an EBD SI canceller that is recognized with its remarkable SI rejection features and small physical size.

By employing a Wi-Fi like waveform, the simulation result has confirmed that the proposed model produces sufficient frequency granularity required for a wide range of wireless sensing applications. Despite the existence of a second party transmitter, the simulation also illustrates that a high-SNR Doppler signal can be extracted in IBFD mode already when the EBD provides $>20 d B$ Tx-Rx isolation at RF.

This paper also explained how the EBD constraints the system performance in both communication and sensing dimensions as it has to be tuned concerning the environmental dynamics resulting in a communication and sensing tradeoff. In this paper, we have illustrated that the packet-wise EBD tuning ensures optimum SI rejection, while it limits the range of the detectable velocities. Because this tuning scheme shortens the Doppler observation window to the duration of the Tx packet.

We also investigated the impact of the target distance on the EBD SI cancelation performance. Regarding the simulation and experimental results, we conclude that when the application involves physical dynamics within $1 \mathrm{~m}$ from the IBFD device, better communication performance can be obtained by a higher level of Tx-Rx isolation and via transmitting shorter packets. As a cost, it narrows down the Doppler velocity range. Hence, use cases which require measurement of slow dynamics, e.g., hand gesture detection integrated into a handheld device, are not feasible in SNR-sensitive scenarios.

Meanwhile, if the application dictates a high level of communication performance, the presented system can detect a broader Doppler velocity range for targets located in larger distances. In such a situation, therefore, a ceiling-mounted platform is more likely to produce a wider range of Doppler velocities, including small speeds.

\section{ACKNOWLEDGMENT}

This work was funded by the European Union's Horizon 2020 under grant agreement no. 732174 (ORCA project). The authors also would like to thank E. Vinogradov for his helpful comments.

\section{REFERENCES}

[1] D. Kim, H. Lee, and D. Hong, "A survey of in-band full-duplex transmission: From the perspective of PHY and MAC layers," IEEE Communications Surveys \& Tutorials, vol. 17, no. 4, pp. 2017-2046, 2015

[2] G. Liu, F. R. Yu, H. Ji, V. C. Leung, and X. Li, "In-band full-duplex relaying: A survey, research issues and challenges," Resource, vol. 147, p. $172,2015$.

[3] T. Vermeulen, F. Rosas, M. Verhelst, and S. Pollin, "Performance analysis of in-band full duplex collision and interference detection in dense networks," in Consumer Communications \& Networking Conference (CCNC), 2016 13th IEEE Annual. IEEE, 2016, pp. 595-601.

[4] X. Zhu and Y. Feng, "RSSI-based algorithm for indoor localization," Communications and Network, vol. 5, no. 02, p. 37, 2013.

[5] X. Luo, W. J. OBrien, and C. L. Julien, "Comparative evaluation of received signal-strength index (RSSI) based indoor localization techniques for construction jobsites," Advanced Engineering Informatics, vol. 25, no. 2, pp. 355-363, 2011.

[6] S. Sigg, U. Blanke, and G. Troster, "The telepathic phone: Frictionless activity recognition from WIFI-RSSI," in Pervasive Computing and Communications (PerCom), 2014 IEEE International Conference on. IEEE, 2014, pp. 148-155.

[7] P. Biswas and Y. Ye, "Semidefinite programming for ad hoc wireless sensor network localization," in Proceedings of the 3rd international symposium on Information processing in sensor networks. ACM, 2004, pp. $46-54$.

[8] K. Wu, J. Xiao, Y. Yi, M. Gao, and L. M. Ni, "Fila: Fine-grained indoor localization," in INFOCOM, 2012 Proceedings IEEE. IEEE, 2012, pp. 2210-2218.

[9] B. Tan, K. Woodbridge, and K. Chetty, "A real-time high resolution passive WiFi doppler-radar and its applications," in Radar Conference (Radar), 2014 International. IEEE, 2014, pp. 1-6.

[10] B. Tan and K. Woodbridge, "A wireless passive radar system for real-time through-wall movement detection," IEEE Transactions on Aerospace and Electronic Systems, vol. 52, no. 5, pp. 2596-2603, 2017.

[11] O. Zhang and K. Srinivasan, "Mudra: User-friendly fine-grained gesture recognition using WiFi signals," in Proceedings of the 12th International on Conference on emerging Networking EXperiments and Technologies. ACM, 2016, pp. 83-96.

[12] Q. Chen, B. Tan, K. Chetty, and K. Woodbridge, "Activity recognition based on micro-doppler signature with in-home Wi-Fi," in e-Health Networking, Applications and Services (Healthcom), 2016 IEEE 18th International Conference on. IEEE, 2016, pp. 1-6.

[13] B. van Liempd, B. Hershberg, S. Ariumi, K. Raczkowski, K.-F. Bink, U. Karthaus, E. Martens, P. Wambacq, and J. Craninckx, "A+ 70dbm iip3 electrical-balance duplexer for highly integrated tunable frontends," IEEE Transactions on Microwave Theory and Techniques, vol. 64, no. 12, pp. 4274-4286, 2016.

[14] S. H. Abdelhalem, P. S. Gudem, and L. E. Larson, "Hybrid transformerbased tunable differential duplexer in a 90-nm CMOS process," IEEE Transactions on Microwave Theory and Techniques, vol. 61, no. 3, pp. 1316-1326, 2013.

[15] D. Tse and P. Viswanath, Fundamentals of wireless communication. Cambridge university press, 2005.

[16] T. Vermeulen, B. van Liempd, B. Hershberg, and S. Pollin, "Real-time RF self-interference cancellation for in-band full duplex," in Dynamic Spectrum Access Networks (DySPAN), 2015 IEEE International Symposium on. IEEE, 2015, pp. 275-276.

[17] A. C. Carusone and D. A. Johns, "Analog filter adaptation using a dithered linear search algorithm," in Circuits and Systems, 2002. ISCAS 2002. IEEE International Symposium on, vol. 4. IEEE, 2002, pp. IV-IV. 